\title{
Highly optimized fourth-order short-time approximation for path integrals
}

\author{
Cristian Predescu* \\ Department of Chemistry and Kenneth S. Pitzer Center for Theoretical Chemistry, \\ University of California, Berkeley, California 94720
}

(Dated: November 14, 2006)

\begin{abstract}
We derive a fourth-order short-time approximation for use in imaginary-time path-integral simulations. The short-time approximation converges for all continuous and bounded from below potentials, attains quartic order of convergence for sufficiently smooth potentials, and utilizes statistically independent random variables for its construction. These properties recommend the approximation as a natural replacement of the trapezoidal Trotter-Suzuki approximation for physical systems with continuous distributions.
\end{abstract}

PACS numbers: 05.30.-d, 02.70.Ss

Keywords: path integrals, short-time approximation, convergence order

With the advent of massively parallel computational architectures, path integral simulations are more and more utilized for the evaluation of quantum statistical properties at a level of accuracy not attainable by other techniques. The now-classical applications for lowtemperature Bosonic systems summarized in Ceperley's decade-old review ${ }^{1}$ are a vivid illustration of the physical and numerical insights brought in by the path integral formalism. Nowadays, the use of path integral simulations as investigation tools is on the increase, whether because the magnitude of the quantum effects is being re-evaluated or because the numerical efficiency has improved to the point that the utilization of more approximate techniques is no longer warranted. ${ }^{2}$ Accordingly, the computational trend is toward the development of general purpose path integral techniques that are robust and efficient enough to be applied for systems of increasing complexity. For those numerical algorithms that are constructed via a Lie-Trotter product, a task of immediate interest is the development of efficient generalpurpose short-time approximations.

In order to appeal to researchers of wide scientific interests, a short-time approximation must satisfy several stringent numerical requirements. As such, it should not depend strongly on the form of the potential function. The amount of pre-computation must be minimal. If possible, the short-time approximation should be constructed in terms of the potential function alone. The resulting Lie-Trotter product should converge for a sufficiently large class of potentials, a class that is normally expected to include the continuous and bounded from below functions. For the resulting path-integral technique to be computationally efficient, the short-time approximation must exhibit a high order of convergence for smooth enough potentials. However, this high convergence order should not cause a significant decrease in the quality of the Monte Carlo sampling. Such a situation might appear when strongly correlated path variables are utilized for the construction of the short-time approximation.

The short-time approximations developed so far attempt to compromise on the aforementioned features.
For example, the Trotter-Suzuki trapezoidal approximation is a functional of the potential (therefore easy to implement) and converges for all continuous and bounded from below potentials. However, it can only attain quadratic convergence, asymptotically. ${ }^{3,4}$ The pair-product approximation considered by Pollock and Ceperley $^{5}$ is numerically stable and has cubic convergence order, but, as its name implies, it only applies for potentials that are sums of two-body terms. The partialaveraging technique ${ }^{6}$ behaves extremely well numerically, converging for a large class of potentials, ${ }^{7}$ but requires the computation of the Gaussian transform of the potential. The Takahashi-Imada ${ }^{8}$ as well as the Suzuki ${ }^{9}$ approximations, may achieve quartic convergence, but require explicit knowledge of the first-order derivatives of the potential and do not converge if these derivatives are not finite. A similar objection can be raised of the short-time approximations recently constructed by Bogojević, Balaž, and Belić ${ }^{10}$ which, while featuring significant improvements in the asymptotic convergence, have the drawback that require explicit knowledge of highorder derivatives of the potential.

In a recent work, ${ }^{11}$ it has been argued that there exist short-time approximations of the form

$$
\begin{array}{r}
\rho_{0}^{(\nu)}\left(x, x^{\prime} ; \beta\right)=\rho_{f p}\left(x, x^{\prime} ; \beta\right) \int_{\mathbb{R}} d \mu\left(a_{1}\right) \ldots \int_{\mathbb{R}} d \mu\left(a_{n_{\nu}}\right) \\
\times \exp \left\{-\beta \sum_{i=1}^{n_{q}} w_{i} V\left[x_{r}\left(\theta_{i}\right)+\sigma \sum_{k=1}^{n_{\nu}} a_{k} \tilde{\Lambda}_{k}\left(\theta_{i}\right)\right]\right\}
\end{array}
$$

that have arbitrary convergence order for sufficiently smooth potentials. In Eq. (1), $\rho_{f p}\left(x, x^{\prime} ; \beta\right)$ is the density matrix of a free particle, $\beta=1 /\left(k_{B} T\right)$ is the inverse temperature, $x_{r}(u)=x+\left(x^{\prime}-x\right) u$ is the reference path, $\sigma=\left(\hbar^{2} \beta / m_{0}\right)^{1 / 2}$, and $d \mu(z)=(2 \pi)^{-1 / 2} \exp \left(-z^{2} / 2\right) d z$ is the standard normal distribution on the real axis $\mathbb{R}$. The positive weights $w_{i}$ and the knots $\theta_{i}$ define a symmetric quadrature rule on the interval $[0,1]$. In order for the short-time approximation to possess the prescribed convergence order $\nu$, the quadrature rule as well as the values $\tilde{\Lambda}_{k}\left(\theta_{i}\right)$ must satisfy certain equations that will be reviewed below. It is useful to regard the quantities $\tilde{\Lambda}_{k}(u)$ 
as continuous functions on $[0,1]$, although only their values on the quadrature knots are relevant. The functions $\tilde{\Lambda}_{k}(u)$ must vanish at the end points of the interval $[0,1]$. Finally, the covariance matrix

$$
\tilde{\gamma}(u, \tau)=u \tau+\sum_{k=1}^{n_{\nu}} \tilde{\Lambda}_{k}(u) \tilde{\Lambda}_{k}(\tau)
$$

must satisfy the identity

$$
\tilde{\gamma}(1-u, 1-\tau)=1-u-\tau+\tilde{\gamma}(u, \tau)
$$

This property ensures that the short-time approximation defined by Eq. (1) is Hermitian. It can be enforced, for example, by searching for functions $\tilde{\Lambda}_{k}(u)$ that are either symmetric or antisymmetric.

Under the hypothesis that the weights $w_{i}$ sum to 1 , the Lie-Trotter products constructed from the short-time approximations of the type given by Eq. (1) converge for all continuous and bounded from below potentials (a proof for the subsequence $n=2^{k}-1$ can be found in Ref. 12). Explicit approximations of order 4 have been already constructed in the literature. ${ }^{11}$ Since then, in unpublished work, the present author has constructed approximations of order 5 , with the purpose of providing numerical evidence that approximations of higher orders do exist. However, for example, the approximation of order 4 utilizes four Gauss-Legendre quadrature knots and three functions $\tilde{\Lambda}_{k}(u)$. Likewise, the approximation of order 5 utilizes five Gauss-Legendre quadrature knots and four functions $\tilde{\Lambda}_{k}(u)$. These additional functions overlap strongly and constitute three or four additional sampling layers in the Lévy-Ciesielski form ${ }^{13}$ of the Lie-Trotter product. If the potential function is not sufficiently smooth and the approximation fails to achieve its highest possible convergence order, then the utilization of 4 or 5 quadrature knots and the fact that the functions $\tilde{\Lambda}_{k}(u)$ overlap strongly constitute a disadvantage in comparison with lower-order approximations that allow for a higher Trotter index at the same computational effort.

In the present work, we shall construct a fourth-order short-time approximation that has the following desirable features that increase the efficiency of the approximation for potentials that are not very smooth: it only utilizes three quadrature knots and two additional functions $\tilde{\Lambda}_{k}(u)$. Moreover, the additional functions are shown to have disjoint support on the set of quadrature knots. Consequently, the functions constitute only one additional sampling layer in the Lévy-Ciesielski form of the Lie-Trotter product. Therefore, the short-time approximation maintains a high sampling efficiency, which is the more important feature when the potential is not sufficiently well-behaved.

The equations that the quadrature rule and the values $\tilde{\Lambda}_{k}\left(\theta_{i}\right)$ must satisfy have been formulated in terms of generalized moments of Gaussian processes ${ }^{11}$ and subsequently reformulated in terms of covariance matrices alone. ${ }^{12} \mathrm{~A}$ short-time approximation of the form given by Eq. (1) has convergence order $\nu$ if and only if the equality of the $\left(\lambda_{0}, \lambda_{1}, \ldots, \lambda_{d}\right)$-polynomials

$$
\begin{gathered}
\int_{0}^{1} d \omega\left(u_{1}\right) \cdots \int_{0}^{1} d \omega\left(u_{d}\right)\left[\sum_{i=0}^{d} \sum_{j=0}^{d} \lambda_{i} \lambda_{j} \tilde{\gamma}\left(u_{i}, u_{j}\right)\right]^{n} \\
=\int_{0}^{1} d u_{1} \cdots \int_{0}^{1} d u_{d}\left[\sum_{i=0}^{d} \sum_{j=0}^{d} \lambda_{i} \lambda_{j} \gamma\left(u_{i}, u_{j}\right)\right]^{n}
\end{gathered}
$$

holds for all $d$ and $n$ such that $d+n=\nu$. Here, $u_{0}=1$, $\gamma(u, \tau)=\min \{u, \tau\}$ is the covariance matrix for a standard Brownian motion, whereas $d \omega(u)$ is a shorthand for the discrete quadrature measure

$$
d \omega(u)=\sum_{i=1}^{n_{q}} w_{i} \delta\left(u-\theta_{i}\right) d u .
$$

The number of functional equations that are generated from this equality of polynomials increases quickly, in an exponential fashion, with the order of convergence $\nu{ }^{11}$ Significant reductions in the number of equations can be obtained by enforcing the equality $\tilde{\gamma}(u, u)=\gamma(u, u)=u$ on the quadrature knots, that is,

$$
\sum_{k=1}^{n_{\nu}} \Lambda_{k}\left(\theta_{i}\right) \Lambda_{k}\left(\theta_{i}\right)=\theta_{i}\left(1-\theta_{i}\right), \quad \forall 1 \leq i \leq n_{q} .
$$

In other words, we require that the discrete Gaussian process utilized in the construction of the short-time approximation matches exactly the variance of the Brownian motion on the quadrature knots. Such an approximation has been dubbed "reweighted." 11 Numerical experiments suggest that this constraint is incompatible with orders of convergence greater or equal to 5 . The unpublished example of order 5 constructed by the author does not satisfy this constraint. Nevertheless, for the convergence order 4 , the constraint leads to a significant reduction in the number of functional equations, which facilitates a very compact solution.

To understand how this reduction appears, consider the case $d=1$ and $n=3$ in Eq. (4). One computes

$$
\begin{aligned}
& \sum_{i=1}^{n_{q}} w_{i}\left[\lambda_{0}^{2}+2 \lambda_{0} \lambda_{1} \tilde{\gamma}\left(1, \theta_{i}\right)+\lambda_{1}^{2} \tilde{\gamma}\left(\theta_{i}, \theta_{i}\right)\right]^{3} \\
& =\int_{0}^{1}\left[\lambda_{0}^{2}+2 \lambda_{0} \lambda_{1} \gamma(1, u)+\lambda_{1}^{2} \gamma(u, u)\right]^{3} d u
\end{aligned}
$$

Since $\tilde{\gamma}(1, u)=\gamma(1, u)=u$ and $\tilde{\gamma}(u, u)=\gamma(u, u)=u$, one sees that Eq. (6) holds true provided that the quadrature scheme specified by the weights $w_{i}$ and knots $\theta_{i}$ integrates exactly all polynomials of degree greater or less than three. Therefore, we require that

$$
\sum_{i=1}^{n_{q}} w_{i}\left(\theta_{i}\right)^{k}=1 /(k+1), \quad \text { for } k=0,1,2,3 \text {. }
$$

Had we not enforced the equality $\tilde{\gamma}(u, u)=u$ on the quadrature knots, the number of resulting equations 
TABLE I: Parameters defining the short-time approximation of order 4 .

\begin{tabular}{|c|c|c|c|}
\hline$i$ & 1 & 2 & 3 \\
\hline$w_{i}$ & $1 / 3$ & $1 / 3$ & $1 / 3$ \\
\hline$\theta_{i}$ & $(2-\sqrt{2}) / 4$ & $1 / 2$ & $(2+\sqrt{2}) / 4$ \\
\hline$\tilde{\Lambda}_{1}\left(\theta_{i}\right)$ & 0 & $1 / 2$ & 0 \\
\hline$\tilde{\Lambda}_{2}\left(\theta_{i}\right)$ & $\sqrt{2} / 4$ & 0 & $\sqrt{2} / 4$ \\
\hline
\end{tabular}

would have been significantly larger. We let it for the reader to demonstrate by explicit computation that all equalities of polynomials implied by Eq. (4) are satisfied provided that the following equations, additional to those expressed by Eq. (7), hold true:

$$
\sum_{i=1}^{n_{q}} \sum_{j=1}^{n_{q}} w_{i} w_{j} \tilde{\gamma}\left(\theta_{i}, \theta_{j}\right)^{k}=\int_{0}^{1} \int_{0}^{1} \gamma(u, \tau)^{k} d u d \tau
$$

for $k=1,2$, and

$$
\sum_{i=1}^{n_{q}} \sum_{j=1}^{n_{q}} w_{i} w_{j} \theta_{i} \tilde{\gamma}\left(\theta_{i}, \theta_{j}\right)=\int_{0}^{1} \int_{0}^{1} u \gamma(u, \tau) d u d \tau .
$$

Remembering the symmetry of the quadrature rule as well as Eq. (3), we see that it is enough to check Eqs. (7) for $k=0,2$ as well as Eqs. (8) for $k=1,2$. The remaining two quadrature equations and Eq. (9) are then automatically satisfied by symmetry. Thus, the final equations read

$$
\begin{aligned}
& \sum_{i=1}^{n_{q}} w_{i}=1, \quad \sum_{i=1}^{n_{q}} \sum_{j=1}^{n_{q}} w_{i} w_{j} \tilde{\gamma}\left(\theta_{i}, \theta_{j}\right)=\frac{1}{3} \\
& \sum_{i=1}^{n_{q}} w_{i}\left(\theta_{i}\right)^{2}=\frac{1}{3}, \quad \sum_{i=1}^{n_{q}} \sum_{j=1}^{n_{q}} w_{i} w_{j} \tilde{\gamma}\left(\theta_{i}, \theta_{j}\right)^{2}=\frac{1}{6} .
\end{aligned}
$$

To obtain the right-hand side values, remember that $\gamma(u, \tau)=\min \{u, \tau\}$ and explicitly compute the righthand side integrals in Eq. (8).

In order to minimize the number of calls to the potential function, we try to construct a solution that has minimal $n_{q}$. No solution exists for $n_{q}=2$. Indeed, the quadrature rule would then be completely specified and equal to the 2-point Gauss-Legendre rule. The covariance matrix $\tilde{\gamma}\left(\theta_{i}, \theta_{j}\right)$ has the two diagonal elements completely determined by Eq. (5). The two off-diagonal terms are equal by symmetry and cannot accommodate both the remaining equations involving the covariance matrix, as it can be shown by explicit computation. Next, we look for $n_{q}=3$. In order to minimize the number of additional Gaussian variables, we try a solution with $n_{\nu}=1$. Unfortunately, no such solution exists. The reader can prove this assertion by observing that the symmetry requirements and Eq. (5) lead to $\tilde{\Lambda}_{1}(u)=[u(1-u)]^{1 / 2}$. This constraint completely specifies the covariance matrix $\tilde{\gamma}\left(\theta_{i}, \theta_{j}\right)$. The remaining quadrature variables $w_{1}, w_{2}$, and $u_{1}$ cannot accommodate the four equalities in Eq. (10).

There are many possible solutions with $n_{q}=3$ and $n_{\nu}=2$. The one we give has the additional property that there are no quadrature knots $\theta_{i}$ such that $\tilde{\Lambda}_{1}\left(\theta_{i}\right)$ and $\tilde{\Lambda}_{2}\left(\theta_{i}\right)$ are both different from zero (that is, the functions $\tilde{\Lambda}_{1}(u)$ and $\tilde{\Lambda}_{2}(u)$ have disjoint support on the set of quadrature knots). The solution is specified by the values from Table I. The verification of Eqs. (10) is left as an exercise for the reader, since it merely involves arithmetic operations. We mention, however, that the present solution is the unique one for which $\tilde{\Lambda}_{1}(u)$ and $\tilde{\Lambda}_{2}(u)$ have disjoint support on the set of quadrature knots. Because of this property, the short-time approximation factorizes as

$$
\begin{aligned}
\rho_{0}^{(4)}\left(x, x^{\prime} ; \beta\right)= & \rho_{f p}\left(x, x^{\prime} ; \beta\right)\left\{\int_{\mathbb{R}}(2 \pi)^{-1 / 2} e^{-a_{1}{ }^{2} / 2} e^{-\beta w_{2} V\left[x_{r}\left(\theta_{2}\right)+\sigma a_{1} \tilde{\Lambda}_{1}\left(\theta_{2}\right)\right]} d a_{1}\right\} \\
& \times\left\{\int_{\mathbb{R}}(2 \pi)^{-1 / 2} e^{-a_{2}{ }^{2} / 2} e^{-\beta w_{1} V\left[x_{r}\left(\theta_{1}\right)+\sigma a_{2} \tilde{\Lambda}_{2}\left(\theta_{1}\right)\right]-\beta w_{3} V\left[x_{r}\left(\theta_{3}\right)+\sigma a_{2} \tilde{\Lambda}_{2}\left(\theta_{3}\right)\right]} d a_{2}\right\} .
\end{aligned}
$$

Any Monte Carlo sampling involving the short-time approximation must proceed in two steps. In the first step, one samples the variables $x$ and $x^{\prime}$ or, rather, the chain that they form in the Lie-Trotter product. In the second step, one keeps the variables $x$ and $x^{\prime}$ fixed and samples the variables $a_{1}$ and $a_{2}$. As Eq. (11) shows, the variables $a_{1}$ and $a_{2}$ are independent and can be tested for acceptance individually. These operations can be performed with a sole evaluation of the action.

In the remainder of the work, we verify numerically the order of convergence of the technique for a sufficiently smooth potential. The physical problem we treat is that of a He atom moving in a double-well potential described by the equation

$$
V(x)=A \cosh (a x)-B \cosh (b x),
$$

with $a=5.79 \AA^{-1}, b=1.518 \AA^{-1}, A=0.121 \mathrm{~K}$, and $B=9.564 \mathrm{~K}$. The parameters are chosen so that to model a physically reasonable one-dimensional cage for the He atom. The mass of the atom is taken to be 


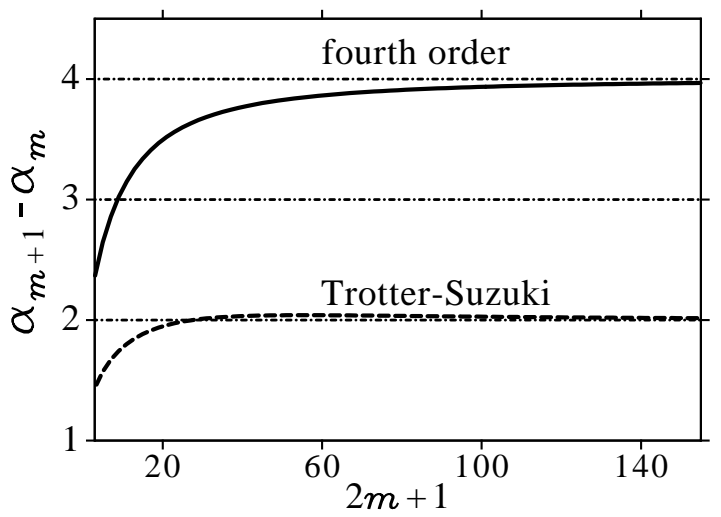

FIG. 1: Numerical demonstration that the convergence order of the short-time approximation given by Eq. (11) is 4, at least for the He cage problem. For comparison, the convergence order of the trapezoidal Trotter-Suzuki approximation is also featured.

$m_{0}=4 \mathrm{amu}$ and the temperature is set to $T=5.11 \mathrm{~K}$. At this temperature, the He atom mostly resides in its quantum groundstate. We employ the matrix multiplication technique to study the convergence order for the partition function. The details of the procedure can be found elsewhere. ${ }^{11}$ The study relies on the fact that the slope $\alpha_{m+1}-\alpha_{m}$ of the sequence

$$
\alpha_{m}=m^{2} \ln \left[1+\frac{Z_{2 m-1}(\beta)-Z_{2 m+1}(\beta)}{Z_{2 m+1}(\beta)-Z(\beta)}\right]
$$

converges to the convergence order in the limit that the Trotter index $2 m+1$ goes to infinity. Here, $Z_{n}(\beta)$ and $Z(\beta)$ represent the partition function computed from the $n$-th order Lie-Trotter product

$$
\begin{array}{r}
\rho_{n}\left(x, x^{\prime} ; \beta\right)=\int_{\mathbb{R}} d x_{1} \ldots \int_{\mathbb{R}} d x_{n} \rho_{0}^{(4)}\left(x, x_{1} ; \frac{\beta}{n+1}\right) \\
\ldots \rho_{0}^{(4)}\left(x_{n}, x^{\prime} ; \frac{\beta}{n+1}\right)
\end{array}
$$

and the exact partition function, respectively. The numerical results presented in Fig. 1 demonstrate that the convergence order of the technique is indeed 4 . When comparing with the trapezoidal Trotter-Suzuki approximation, we see that the maximal convergence order is attained for relatively large Trotter indexes. This trend demonstrates that improving the convergence orders indefinitely may not necessarily lead to spectacular numerical gains. The reason is that systematic errors comparable to the statistical Monte Carlo errors may be achieved for relatively low Trotter indexes, for which the maximal convergence order is not yet attained.

To summarize, the short-time approximation considered in the present work satisfies most of the requirements enumerated in the introductory paragraphs. As such, it is convergent for all continuous and bounded from below potentials and, provided that the potential function is sufficiently smooth, achieves quartic convergence. Its construction is realized solely in terms of the potential function, which makes the approximation numerically appealing. The two additional Gaussian variables utilized in the construction can be sampled independently and simultaneously in a Monte Carlo simulation. We would like to emphasize that the stringent requirements in the second paragraph are consistent with the general expectations in chemistry, where the potentials necessarily contain dominant three-body terms and are sufficiently complex to render the use of derivatives or explicit Gaussian transforms a tough computational task. Nevertheless, for certain physical applications with less-complex potential functions, some of the path-integral techniques mentioned in the introductory paragraphs might prove to be a computationally more efficient alternative. Also, one could envision scenarios where the two-body part of the potential function is handled by means of the pairproduct approximation, whereas the remaining manybody part, which is a smooth function, is treated by means of the fourth-order approximation.

Sure enough, higher-order short-time approximations are desirable, but future work will have to weigh the gains against the possible loss of Monte Carlo sampling efficiency. Most likely, higher-order approximations exhibit a strong overlap of the functions $\tilde{\Lambda}_{k}(u)$ on the quadrature knots, property that diminishes the efficiency of Monte Carlo samplers. Again, we are thinking of those situations where the derivatives of the potential function are either not defined or poorly behaved, as magnitude. In such cases, the higher convergence order does not help much. Nevertheless, the superior efficiency of the Monte Carlo samplers facilitated by the lack of overlap of the functions $\tilde{\Lambda}_{k}(u)$ on the quadrature knots is always guaranteed, whether the potential is smooth or not.

The present development relies heavily on the availability of a Feynman-Kac formula. It can in principle be extended to some other situations where the potential might be temperature dependent (perhaps as a result of integrating out some bath variables) or for stochastic processes in continuous time for which a drifting term is present, such as the Smoluchovski equation. Although we have kept the nomenclature of Lie-Trotter product, the convergence of the ensuing product form does not follow from the Trotter theorem, but is a consequence of the Feynman-Kac formula. ${ }^{12}$ On the other hand, for the situations where a Feynman-Kac formula is not available, the present approach cannot be applied. Examples of such situations are provided by the real-time propagation or by the statistical mechanics of those systems that are essentially quantum-mechanical and for which no classical Hamiltonian is available. ${ }^{14}$ It is quite intriguing to notice that in these latter situations one can construct generalized forms of the Lie-Trotter exponential product that exhibit high convergence orders, whether because the short-time approximations themselves are bounded ${ }^{15,16}$ or because the non-comuting operators making up the 
Hamiltonian are bounded. ${ }^{17}$

\section{Acknowledgments}

This work was supported in part by the National Science Foundation Grant No. CHE-0345280 and by the
Director, Office of Science, Office of Basic Energy Sciences, Chemical Sciences, Geosciences, and Biosciences Division, U.S. Department of Energy under Contract No. DE-AC02-05CH11231.
* Electronic address: cpredescu@comcast.net

${ }^{1}$ Ceperley, D. M. Rev. Mod. Phys. 1995, 67, 279.

2 Doll, J. D.; Eleftheriou, M.; Corcelli, S. A.; Freeman, D. L., in Quantum Monte Carlo Methods in Physics and Chemistry, M. P. Nightingale, C. J. Umrigar, eds.; NATO ASI Series, Series C Mathematical and Physical Sciences, Vol. X, Kluwer: Dordrecht, 1999.

3 Suzuki, M. J. Math. Phys., 1985, 26, 601; J. Stat. Phys., 1986, 43,883 .

4 Ichinose T.; Tamura, H. Commun. Part. Diff. Eq., 2004, 29, 1905.

5 Pollock, E. L.; Ceperley, D. M. Phys. Rev. B, 1984, 30, 2555.

6 Doll, J. D.; Coalson, R. D.; Freeman, D. L. Phys. Rev. Lett., 1985, 55, 1.
7 Predescu, C. J. Math. Phys. 2003, 44, 1226.

8 Takahashi M.; Imada, M. J. Phys. Soc. Jpn., 1984, 53, 3765.

9 Suzuki, M. Phys. Lett. A 1995, 201, 425.

10 Bogojević, A.; Balaž, A.; Belić, A. Phys. Rev. Lett. 2005, 94, 180403.

11 Predescu, C. Phys. Rev. E 2004, 69, 056701.

12 Predescu, C. math-ph/0508017.

13 Predescu, C. Phys. Rev. E 2005, 71, 045701(R).

14 Suzuki, M. Prog. Theor. Phys. 1976, 56, 1454.

15 Yoshida, H. Phys. Lett. A 1990, 150, 262.

16 Suzuki, M. J. Math. Phys. 1991, 32, 400.

17 Suzuki, M. J. Math. Phys. 1993, 34, 4892. 